\title{
Quantification in Hyperpolarized NMR
}

\section{Supporting Information}

\author{
Arnab Dey and Narayanan Chandrakumar*
}

MRI-MRS Centre, Department of Chemistry, Indian Institute of Technology-Madras

Chennai-600036, Tamil Nadu, India

\section{Derivation of relation between the real enhancement $A_{\mathrm{r}}$ and the measured apparent enhancement $A_{\mathrm{a}}$}

For the signal $S$, we have, given the NMR coil $Q$-factor $Q^{\prime}$ and $Q$, respectively on resonance and far off resonance:

$$
\begin{aligned}
& S=a Q^{\prime} M \sim a Q^{\prime} \chi^{\prime \prime}=a Q^{\prime} \chi_{0} \frac{v_{0}}{\Delta v_{1 / 2}} \\
& Q=\left(\omega_{0} L_{0} / R_{0}\right) .
\end{aligned}
$$

Here, $a$ is a proportionality constant and $M$, the transverse spin magnetization; $Q^{\prime}$ is the $Q$-factor on resonance.

For the coil impedance $Z$, we have in SI units, including the rf spin susceptibility:

$$
Z=i \omega_{0} L_{0}\left(1+\phi \chi^{\prime}-i \phi \chi^{\prime \prime}\right)+R_{0}=i \omega_{0} L_{0}+\omega_{0} L_{0} \phi \chi^{\prime \prime}+R_{0}
$$

Recall that $\chi^{\prime}=0$ on resonance; $\phi$ is the filling factor. 
We now distinguish the $Q$ factor on resonance (denoted $Q^{\prime}$ above) under conditions of equilibrium polarization (denoted $Q_{\mathrm{B}}$ ) and enhanced polarization (denoted $Q_{\mathrm{en}}$ ), with spin susceptibilities $\chi_{B}^{\prime \prime}$ and $\chi_{e n}^{\prime \prime}$ respectively. We have:

$$
\begin{aligned}
& Q_{B}=\frac{\omega_{0} L_{0}}{\left(R_{0}+\omega_{0} L_{0} \phi \chi_{B}^{\prime \prime}\right)}=\frac{Q}{\left(1+Q \phi \chi_{B}^{\prime \prime}\right)} \\
& Q_{e n}=\frac{\omega_{0} L_{0}}{\left(R_{0}+\omega_{0} L_{0} \phi \chi_{e n}^{\prime \prime}\right)}=\frac{Q}{\left(1+Q \phi \chi_{e n}^{\prime \prime}\right)} \\
& S_{B} \sim a Q_{B} \chi_{B}^{\prime \prime}=a \frac{Q}{\left(1+Q \phi \chi_{B}^{\prime \prime}\right)} \chi_{B}^{\prime \prime} \\
& S_{e n} \sim a Q_{e n} \chi_{e n}^{\prime \prime}=a \frac{Q}{\left(1+Q \phi \chi_{e n}^{\prime \prime}\right)} \chi_{e n}^{\prime \prime} \\
& \chi_{e n}^{\prime \prime}=\chi_{B}^{\prime \prime}(A+1)
\end{aligned}
$$

We thus find:

$$
A_{a}+1=\frac{S_{e n}}{S_{B}}=\frac{Q_{e n} M_{e n}}{Q_{B} M_{B}}=\frac{Q_{e n} \chi_{e n}^{\prime \prime}}{Q_{B} \chi_{B}^{\prime \prime}}=\frac{\left(1+Q \phi \chi_{B}^{\prime \prime}\right)}{\left(1+Q \phi \chi_{e n}^{\prime \prime}\right)} \frac{\chi_{e n}^{\prime \prime}}{\chi_{B}^{\prime \prime}}
$$

This leads to:

$$
\begin{aligned}
& A_{a}+1=\frac{\left(1+Q \phi \chi_{B}^{\prime \prime}\right)}{\left(1+Q \phi \chi_{e n}^{\prime \prime}\right)} \frac{\chi_{B}^{\prime \prime}\left(A_{r}+1\right)}{\chi_{B}^{\prime \prime}}=\frac{\left(1+Q \phi \chi_{B}^{\prime \prime}\right)}{\left(1+Q \phi \chi_{e n}^{\prime \prime}\right)}\left(A_{r}+1\right) \\
& \Rightarrow A_{a}+1=\frac{\left(1+Q \phi \chi_{B}^{\prime \prime}\right)}{\left(1+Q \phi \chi_{B}^{\prime \prime}\left(A_{r}+1\right)\right)}\left(A_{r}+1\right)
\end{aligned}
$$


Rearranging, we find:

$$
A_{r}=A_{a} \frac{\left(1+Q \phi \chi_{B}^{\prime \prime}\right)}{\left(1-A_{a} Q \phi \chi_{B}^{\prime \prime}\right)}
$$

Here, the imaginary part of the rf susceptibility on resonance for a spin- $1 / 2$ ensemble may be expressed in the high temperature approximation as:

$$
\chi_{B}^{\prime \prime}=\frac{N \gamma^{2} \hbar^{2}}{4 k T} \frac{v_{0}}{\Delta v_{1 / 2}}
$$

Similarly, we find:

$$
A_{a}=\frac{A_{r}}{\left(1+Q \phi \chi_{B}^{\prime \prime}\left(A_{r}+1\right)\right)}
$$

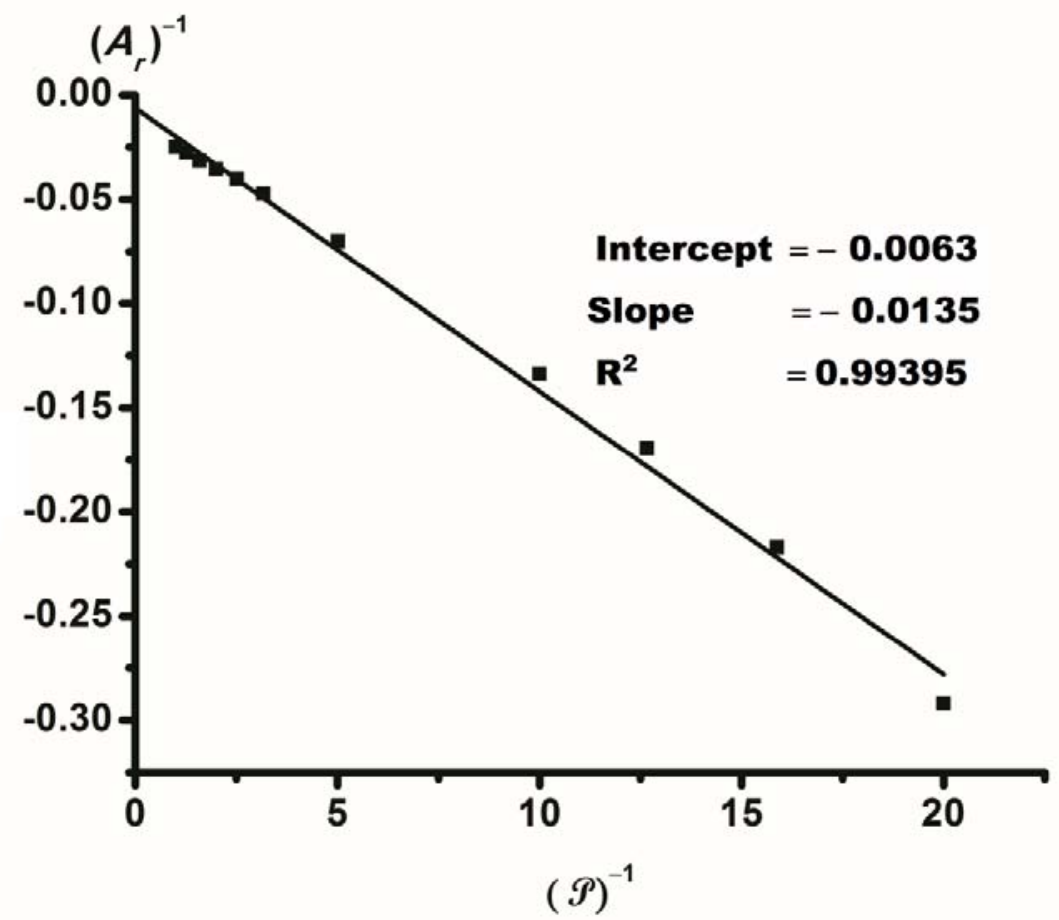

Figure S1. Plot of reciprocal real enhancement $\left(A_{r}\right) v s$. reciprocal relative microwave power $(\mathcal{P})$; system: $\mathrm{C}_{6} \mathrm{H}_{6} /$ Galvinoxyl. 


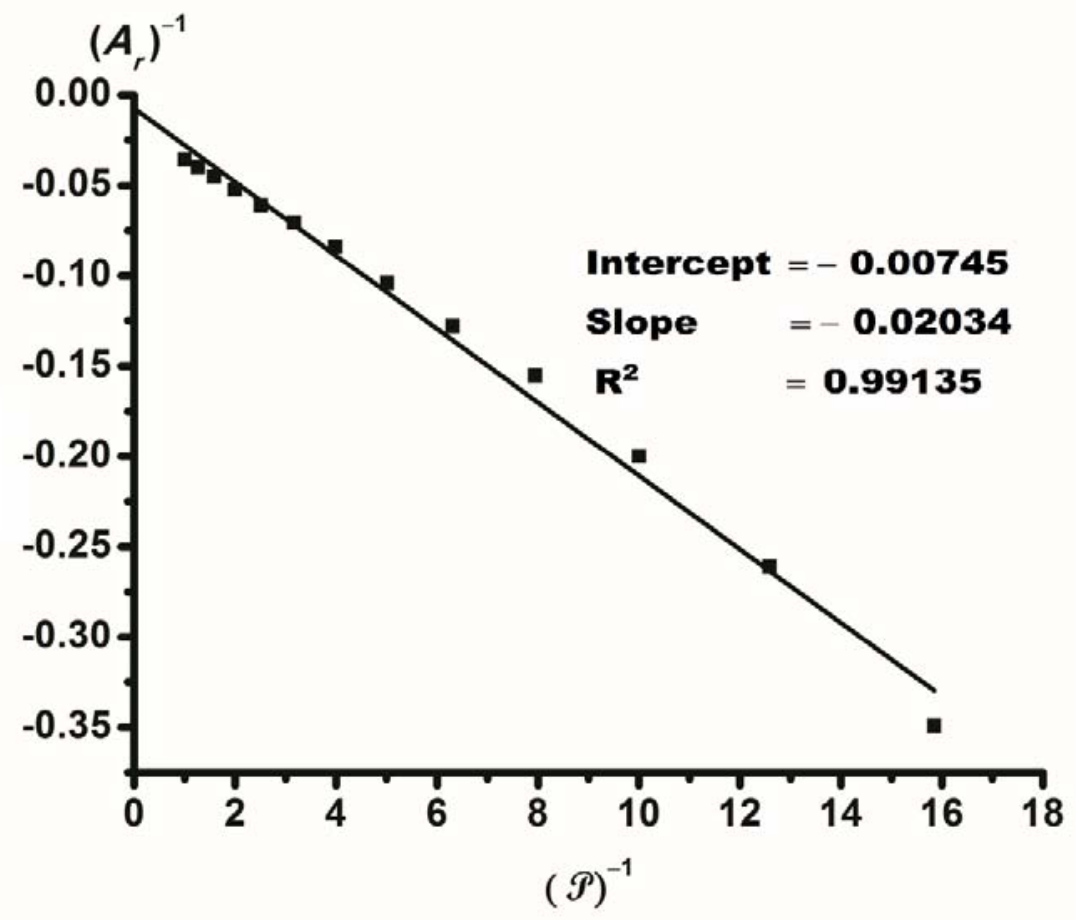

Figure S2. Plot of reciprocal real enhancement $\left(A_{r}\right) v s$. reciprocal relative microwave power $(\mathcal{P})$; system: $\mathrm{C}_{6} \mathrm{H}_{6} / \mathrm{BDPA}$.

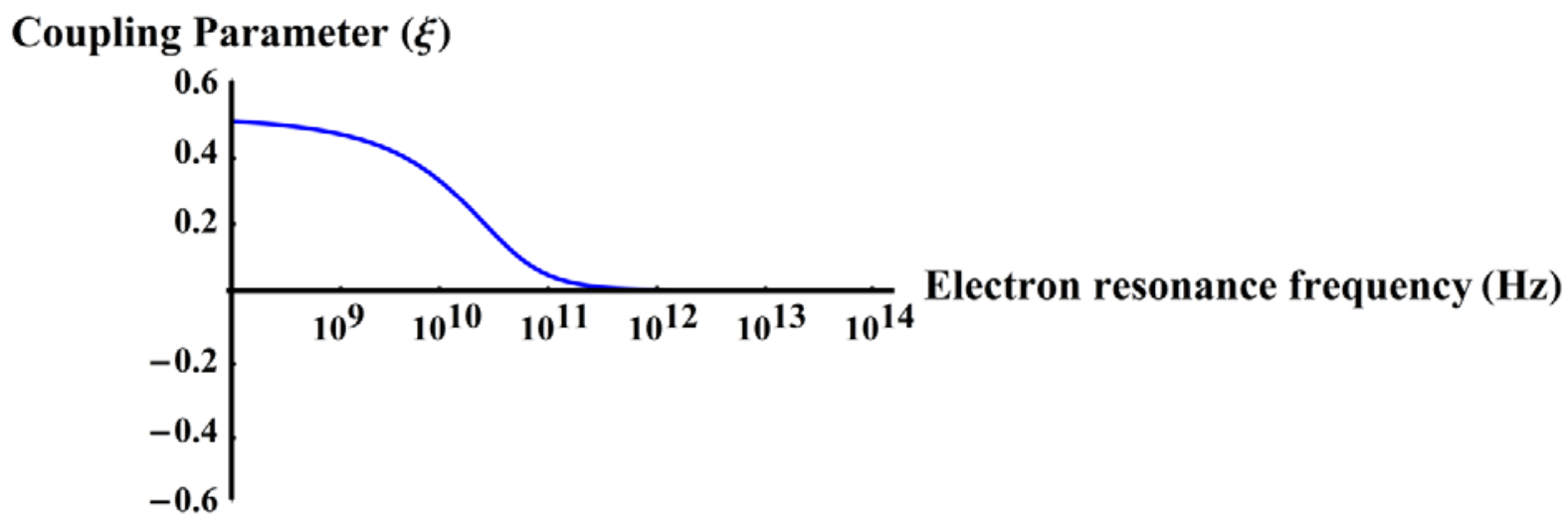

Figure S3. Plot of Coupling Parameter vs. Electron Spin Resonance Frequency at fixed correlation time $4.064 \times 10^{-11} \mathrm{~s}$ (considering dipolar interaction with translational motion).

From this plot we obtain the coupling parameter $(\xi)$ value of 0.3 at an electron resonance frequency of $9.6 \mathrm{GHz}$ (X-Band). The theoretical Ultimate-Ultimate Enhancement value $\left(U_{\infty}\right)$ will thus be 197.4: $\boldsymbol{U}_{\infty}=-(658.2 \times \xi)$. 
Table S1: Real enhancement $A_{\mathrm{r}} v s$. Spin Count for $A_{\mathrm{a}}=-100$

\begin{tabular}{|l|l|l|l|}
\hline Spin Concentration & Spin Count & $A_{r}$ & $A_{a}$ \\
\hline $1 \mathrm{nM}$ & $3.0115 \times 10^{11}$ & -99.99998 & -100 \\
\hline $10 \mathrm{nM}$ & $3.0115 \times 10^{12}$ & -99.99976 & -100 \\
\hline $100 \mathrm{nM}$ & $3.0115 \times 10^{13}$ & -99.99758 & -100 \\
\hline $1 \mu \mathrm{M}$ & $3.0115 \times 10^{14}$ & -99.97579 & -100 \\
\hline $10 \mu \mathrm{M}$ & $3.0115 \times 10^{15}$ & -99.75842 & -100 \\
\hline $100 \mu \mathrm{M}$ & $3.0115 \times 10^{16}$ & -97.63616 & -100 \\
\hline $1 \mathrm{mM}$ & $3.0115 \times 10^{17}$ & -80.54286 & -100 \\
\hline $10 \mathrm{mM}$ & $3.0115 \times 10^{18}$ & -29.72795 & -100 \\
\hline $100 \mathrm{mM}$ & $3.0115 \times 10^{19}$ & -4.88827 & -100 \\
\hline $1 \mathrm{M}$ & $3.0115 \times 10^{20}$ & -1.40307 & -100 \\
\hline $10 \mathrm{M}$ & $3.0115 \times 10^{21}$ & -1.04046 & -100 \\
\hline $100 \mathrm{M}$ & $3.0115 \times 10^{22}$ & -1.00405 & -100 \\
\hline
\end{tabular}

Table S2: Real enhancement $A_{\mathrm{r}} v s$. Spin Count for $A_{\mathrm{a}}=+100$

\begin{tabular}{|l|l|l|l|}
\hline Spin Concentration & Spin Count & $A_{r}$ & $A_{a}$ \\
\hline $1 \mathrm{nM}$ & $3.0115 \times 10^{11}$ & +100.00002 & +100 \\
\hline $10 \mathrm{nM}$ & $3.0115 \times 10^{12}$ & +100.00025 & +100 \\
\hline $100 \mathrm{nM}$ & $3.0115 \times 10^{13}$ & +100.00247 & +100 \\
\hline $1 \mu \mathrm{M}$ & $3.0115 \times 10^{14}$ & +100.02471 & +100 \\
\hline $10 \mu \mathrm{M}$ & $3.0115 \times 10^{15}$ & +100.24766 & +100 \\
\hline $100 \mu \mathrm{M}$ & $3.0115 \times 10^{16}$ & +102.53253 & +100 \\
\hline $1 \mathrm{mM}$ & $3.0115 \times 10^{17}$ & +132.70614 & +100 \\
\hline $10 \mathrm{mM}$ & $3.0115 \times 10^{18}$ & -70.84201 & +100 \\
\hline $100 \mathrm{mM}$ & $3.0115 \times 10^{19}$ & -5.30498 & +100 \\
\hline $1 \mathrm{M}$ & $3.0115 \times 10^{20}$ & -1.41459 & +100 \\
\hline $10 \mathrm{M}$ & $3.0115 \times 10^{21}$ & -1.04131 & +100 \\
\hline $100 \mathrm{M}$ & $3.0115 \times 10^{22}$ & -1.00413 & +100 \\
\hline
\end{tabular}


Table S3: Apparent enhancement $A_{\mathrm{a}} v s$. Spin Count for $A_{\mathrm{r}}=+100$

\begin{tabular}{|l|l|l|l|}
\hline Spin Concentration & Spin Count & $\mathrm{A}_{\mathrm{r}}$ & $\mathrm{A}_{\mathrm{a}}$ \\
\hline $1 \mathrm{nM}$ & $3.0115 \times 10^{11}$ & +100 & +99.99998 \\
\hline $10 \mathrm{nM}$ & $3.0115 \times 10^{12}$ & +100 & +99.99975 \\
\hline $100 \mathrm{nM}$ & $3.0115 \times 10^{13}$ & +100 & +99.99753 \\
\hline $1 \mu \mathrm{M}$ & $3.0115 \times 10^{14}$ & +100 & +99.9753 \\
\hline $10 \mu \mathrm{M}$ & $3.0115 \times 10^{15}$ & +100 & +99.75355 \\
\hline $100 \mu \mathrm{M}$ & $3.0115 \times 10^{16}$ & +100 & +97.58899 \\
\hline $1 \mathrm{mM}$ & $3.0115 \times 10^{17}$ & +100 & +80.18877 \\
\hline $10 \mathrm{mM}$ & $3.0115 \times 10^{18}$ & +100 & +28.81367 \\
\hline $100 \mathrm{mM}$ & $3.0115 \times 10^{19}$ & +100 & +3.89018 \\
\hline $1 \mathrm{M}$ & $3.0115 \times 10^{20}$ & +100 & +0.40313 \\
\hline $10 \mathrm{M}$ & $3.0115 \times 10^{21}$ & +100 & +0.04046 \\
\hline $100 \mathrm{M}$ & $3.0115 \times 10^{22}$ & +100 & +0.00405 \\
\hline
\end{tabular}

